\title{
1 A short remark on Ontogenetic model and its analytical solution
}

2 Ya Li, Ji-Huan He

3

$4 \quad$ National Engineering Laboratory for Modern Silk, College of Textile and Clothing

5 Engineering, Soochow University, 199 Ren-Ai Road, Suzhou 215123, China

6 and

$7 \quad$ Nantong Textile Institute, Soochow University, Nantong, China

8

9 Email: hejihuan@suda.edu.cn

\section{Abstract}

12 The ontogenetic growth modeling has been of conspicuously scientific interest, as well as its practical importance in many industrial activities for optimal design. Much work has been done recently to understand the growth mechanism of living organism with a nonlinear time dependence. Some quantitative metabolic models which have been provided to describe organism growth curves are in comparison with what for ontogenetic growth represented by G.B. West et al. (West et al., 2001) in his basic formulations used to discuss the application on different growth patterns. Herein the analytical solution is also involved. In this paper we re-analyzed the assumptions underlying them to find whether the basic relation of organism growth estimated can inosculate with the equation we have proposed. 
1 Keywords: ontogenetic growth model, mass - relative to the time, differentiate

2 the implicit function, nonlinear dependence

3

$4 \quad$ Introduction

5 As the individual's body size increases, its mass - relative to the time changes. There

6 are so many biological processes from cellular metabolism to organism dynamics and

7 the relationships between size and rate are characterized by particular growth rhythm.

8 More physiological approaches to describe life growth curves have drawn on more

9 sophisticated models for ontogenetic growth.

10 The von Bertalanffy equation used to describe the ontogenetic growth of

11 organisms until West et al. (West et al., 2001) provided a model to describe the

12 ontogenetic growth. The dependence of a biological variable $\mathrm{B}$ on body mass $\mathrm{M}$ is

13 typically characterized by nonlinear system of equations. The base metabolic rate B

14 of numerous species (both warm blooded and cold-blooded organisms) is empirically

15 observed to be proportional to body mass $m$ or volume with an exponent of 0.75 , i.e.

$16 \mathrm{~B}=\mathrm{B}_{0} \mathrm{~m} 3 / 4$ (West et al., 2001), where $\mathrm{B}_{0}$ is a constant that is characteristic of the type

17 of organism. Similarly, the advantages of the logistic model over the ontogenetic

18 growth are the wide adaptability for many species including animal and plant growth

19 (Shi et al., 2013). Obviously, many contending models emerged describing the

20 relationship between mass and time (Shi et al., 2013). Among these models, the von

21 Bertalanffy equation might be the most popular one (Bertalanffy, 1957). Traditionally,

these variables have been related to a single, easily measurable biological attribute: 
1 body size (Calder, 1984). A quantitative model has also been developed to describe

2 ontogenetic growth, taking into account a single average cell in the building block,

3 then the rate equations for $\mathrm{a}, \mathrm{b}$ and $\mathrm{m}$ concentrations can be written as:

4

$5 \quad \frac{d m}{d t}=a m^{\frac{8}{4}}-b m$

6 where the constants a and b can be related to physical properties of the single

7 average cell, $\mathrm{m}$ represents the body mass at time t. It claimed that 0.75 exponent had

8 been well supported by the data on many biological spcies like mammals (Rogers et

9 al., 1995), birds (Weathers and Siegel, 1995), fish (Xie and Sun, 1990; Brett, 1989),

10 mollusks (Hamburger, et al., 1983), chicken (Nagai, et al., 2015) and plants (Enquist,

11 et al., 1998). Then P.J. Shi et.al. tested the generality of the ontogenetic growth on

12 different crop species and compared the goodness-of-fits between the ontogenetic

13 growth and other non-nonlinear models that are often used to describe the ontogenetic

14 growth (Shi et al., 2013). However, they noticed some ignorance of crops existed. In

15 this work we have derived a theoretical framework for the growing trajectory in the

16 context to elucidate the growth of biological species.

17 Materials and methods

\section{Data}

The body mass data of several spcies were extracted from the research of the general model by West et al. and $\mathrm{m}_{0}$ is the intial mass at birth $(\mathrm{t}=0), \mathrm{M}$ represents the

21 asymptotic maximum body mass, which is ultimate constant. They can be written as:

$m(0)=m_{0},(2)$ 
$1 \quad m(\infty)=m_{\infty}=(a / b)^{4},(3)$

2 While these metabolic models provide a valuable insight into ontogenetic growth,

3 further refinements must capture the relevant relation in the architecture of biological

4 species. Ultimately, ontogenetic growth models must also include terms to describe

5 the increase in mass associated with time.

6 Values of $\mathrm{a}, \mathrm{m}_{0}$ and $\mathrm{M}$ for these and several other species had been provided

7 during these research. They mostly explained the sigmoidal time dependence of the

8 mass of the biomass (Ulmschneider and Searson, 2015). Analysis of ontogenetic

9 growth and in vivo data of animal models and humans had been used to suggest that

10 the growth follows a universal growth law with three adjustable parameters: the initial

11 and final mass of the living beings, and the constant $a$, whereas $b\left(=a / M^{1 / 4}\right)$ should

12 scale as $\mathrm{aM}^{-1 / 4}$. The current challenge in this field is to bring physical insight into this

13 universal behavior by combining models (West, 1997).

\section{Models}

15 Consistent with our predictions, we will verify the equations to see whether we 16 gave which had been arranged as Eq.(7) is valid when compared to match if it can fit

17 the biological species. Here, what all the parameters represents have been referred above. Consequently, the derivative of the functions can be got step by step as the following formulas. To differentiate the implicit function of Eq. (1) as

$$
m^{r^{7}}=\frac{3}{4} a \frac{1}{m_{4}^{2}} m^{\gamma}-b m^{\gamma}=\frac{3}{4} a^{2} m^{\frac{1}{2}}+b^{2} m-\frac{7}{4} a b m^{\frac{8}{4}}
$$

21 with intial conditions of Eq. (1) and Eq. (4): 
1

$m^{\prime \prime}(n)=\frac{3}{4} a^{2} m_{0}^{\frac{1}{2}}+b^{2} m_{0}-\frac{\pi}{4} a b m_{0} \frac{8}{4}$,
2

$$
\beta_{1}=\frac{a m_{0}^{3 / 4}-b m_{0}}{m_{\infty} \alpha}=\frac{a m_{0}^{3 / 4}-b m_{0}}{m_{\infty}-m_{0}},(14)
$$


1

$$
\beta_{2}=\frac{\frac{3}{4} a^{2} m_{2}^{\frac{1}{2}} m_{w}+\frac{1}{4} a^{2} m_{0}^{\frac{8}{2}}-\frac{1}{4} a b m_{0}^{\frac{7}{4}}+b^{2} m_{w} m_{0}-\frac{7}{4} a b m_{0}^{\frac{8}{4}} m_{w}}{2\left(m_{\infty}-m_{0}\right)^{2}}
$$

2

3

4

5

6

7

8

9

\section{Relationship between two models}

Briefly, in this work we consider classical methods of ontogenetic growth together

with mixtures theory with a rigid individual. The examples in general equations, were

gave as the figures as below. Besides, The data was chosen from the samples of

7 general equations by West et al. (West et al., 2001). In order to prove the relation with

mass and time, we have set out to determine whether the equation is suitable for the

nonlinear dependence.

0 Put the data in he equations we gave, then we found that all can fit the data extremely well (Fig. 1, Fig. 2, Fig. 3 and Fig.4) by using Eq. (7) .

(1) Guinea pig: $m_{0}=5, \mathrm{M}=840, \mathrm{a}=0.2, \mathrm{~b}=0.04, \alpha=0.99, \beta_{1}=0.000578, \beta_{2}=0.0000167$

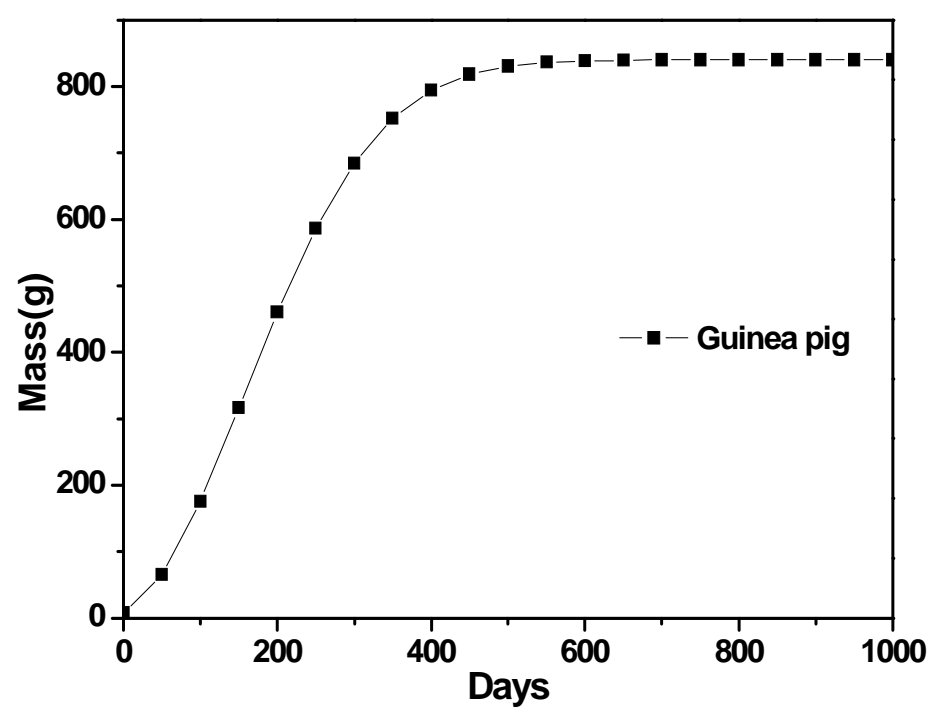

13

14 Fig. 1. Guinea pig typical example of fits to growth curve (solid lines) using Eq. (7). 
1 (2) Guppy : $m_{0}=0.008, \mathrm{M}=0.15, \mathrm{a}=0.1, \mathrm{~b}=0.167, \alpha=0.947, \beta_{1}=0.01, \beta_{2}=0.00045$ 2

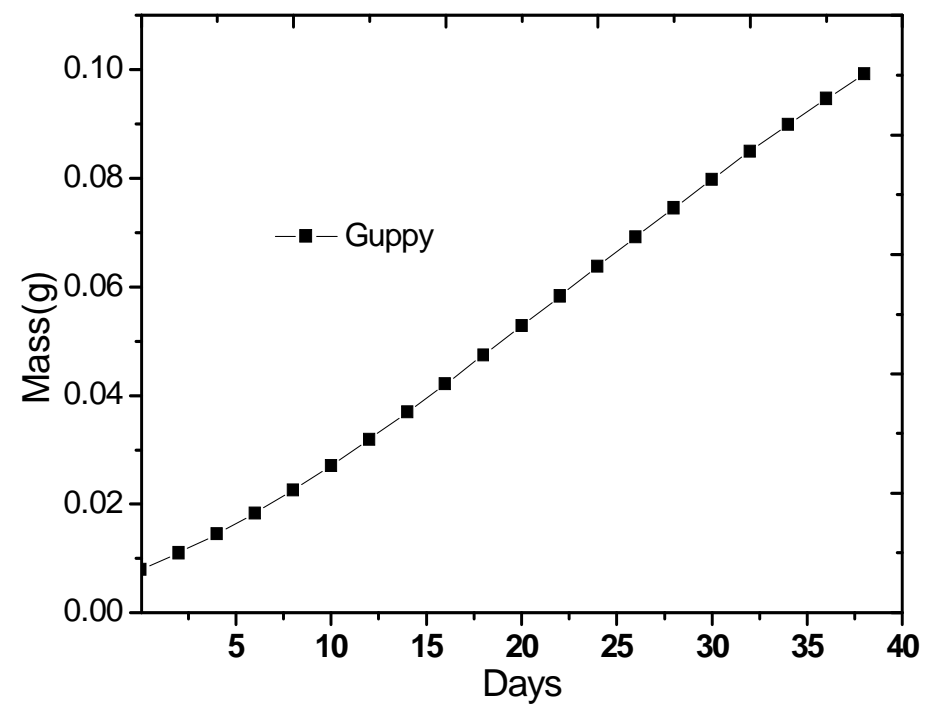

3

4 Fig. 2. Guppy typical example of fits to growth curve (solid lines) using Eq. (7).

5 (3) Hen: $m_{0}=43, \mathrm{M}=2050, \mathrm{a}=0.502, \mathrm{~b}=0.075, \alpha=0.979, \beta_{1}=0.0026, \beta_{2=}=0.0000366$

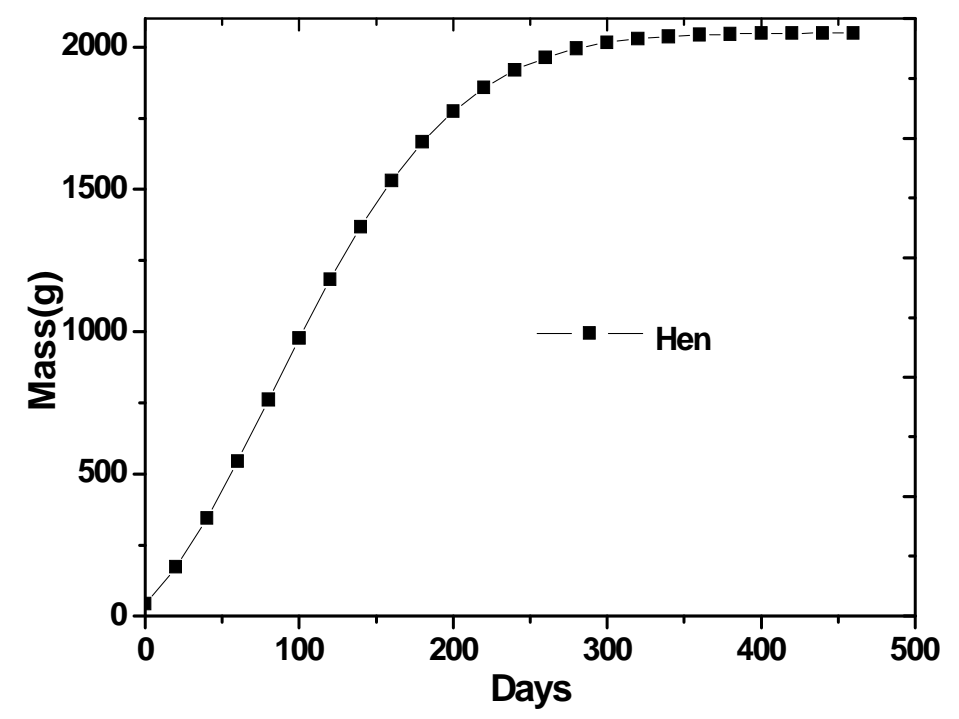

6

$7 \quad$ Fig. 3. Hen example of fits to growth curve (solid lines) using Eq. (7).

8 (4) Cow, $m_{0}=33333, \mathrm{M}=442000, \mathrm{a}=0.276, \mathrm{~b}=0.01, \alpha=0.925, \beta_{1}=0.00085$, 
$1 \quad \beta_{2}=0.00000262$

2

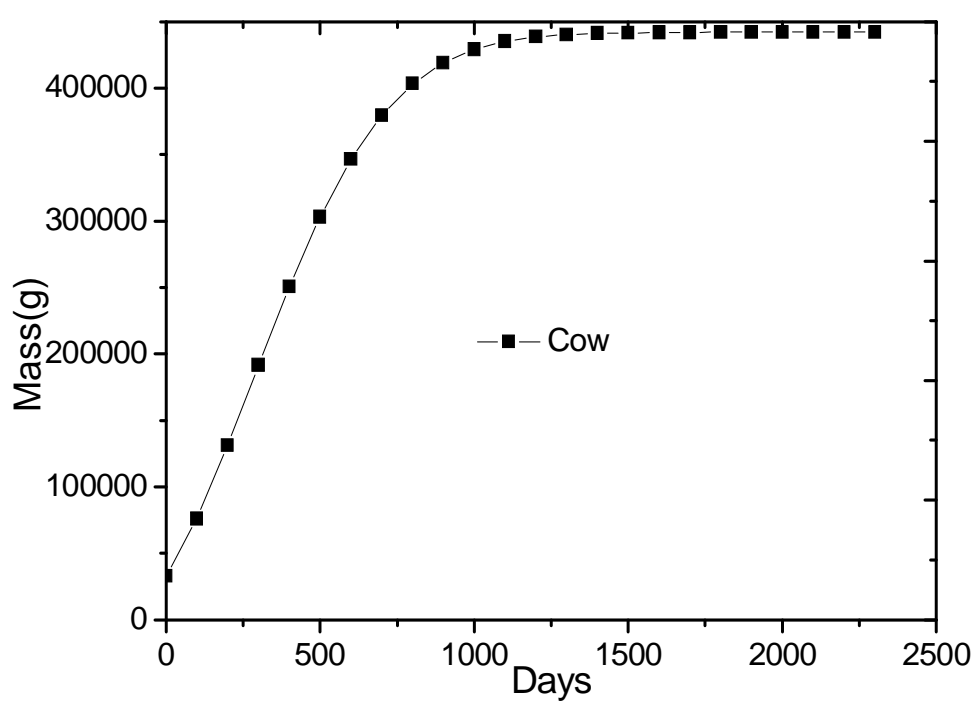

3

4 Fig.4. Cow typical example of fits to growth curve (solid lines) using Eq. (7).

5

\section{Conclusion}

7 In summary, the equation of the mode we gave meets all the samples used in the

8 so-called "general" ontogenetic growth model and they all satisfy the nonlinear

9 dependence. The model structure is simple and typical, it has wide adaptability for

10 many species including animals and crops growth. We get the same shape of the

11 general growth equation from nature.

\section{Acknowledgments}

14 The work is supported by Priority Academic Program Development of Jiangsu

15 Higher Education Institutions (PAPD), National Natural Science Foundation of China

16 under grant No.11372205 and Project for Six Kinds of Top Talents in Jiangsu

17 Province under grant No. ZBZZ-035, Science \& Technology Pillar Program of 
$1 \quad$ Jiangsu Province under grant No. BE2013072.

2

\section{References}

4 West, G.B., Brown, J.H. and Enquist, B.J. (2001). A general model for ontogenetic

$5 \quad$ growth, Nat. 413, 628-631.

6 Shi, P.J., Men, X.Y., Sandhu, H.S., Chakraborty, A., Li, B.L., Fang, O.Y., Sun,

7 Y.C. and Feng, G. (2013). The "general" ontogenetic growth model is

8 inapplicable to crop growth, Ecol. Model. 266, 1-9.

9 Bertalanffy, V.L. (1957). Quantitative laws in metabolism and growth, Q. Rev. Biol.

$10 \quad 32,217-231$.

11 Calder, W.A. (1984). Size, Function, and Life History, Harvard Univ. UK :

12 Cambridge University Press.

Rogers, D. M., Olson, B. L. and Wilmore, J. H. (1995). Scaling for the $\mathrm{V}_{\mathrm{O} 2}$-to-body

14 size relationship among children and adults, J. Appl. Physiol. 79, 958-967 .

15 Weathers, W.W., Siegel, R. B. (1995). Body size establishes the scaling of avian

16 postnatal metabolic rate: an interspecific analysis using phylogenetically

$17 \quad$ independent contrasts, Ibis $\mathbf{1 3 7}, 532-542$.

18 Xie, X.J., Sun, R.Y. (1990). The bioenergetics of the southern catfish (Silurus

19 meridionalis chen). I.Resting metabolic rate as a function of body weight and

20 temperature, Physiol. Zool. 63, 1181-1195.

21 Brett, J.R. (1989). The relation of size to rate of oxygen consumption and sustained

22 swimming speed of Sockeye Salmon (Oncorhynchus nerka), J. Fish Res. Bd Can. 
1

2 Hamburger, K., Møhlenberg, F., Randløv, A. and Riisgård, H.U. (1983). Size,

3

4

5

6

22, 1491-1501.

oxygen consumption and growth in the mussel Mytilus edulis, Mar. Biol. 75, 303-306.

5 Nagai, H., Sezaki, M., Kakiguchi, K., Nakaya, Y., Lee, H.C., Ladher, R.,

6 Sasanami, T., Han, J.Y ., Yonemura, S. and Sheng, G.j. (2015). Cellular

analysis of cleavage-stage chick embryos reveals hidden conservation in

vertebrate early development, Development 142, 1279-1286.

Enquist, B. J., Brown, J. H., West, G. B. (1998). Scaling of plant energetics and population density, Nat. 395, 163-165.

Ulmschneider, M.B., Searson, P.C. (2015). Mathematical models of the steps involved in the systemic delivery of a chemotherapeutic to a solid tumor: From circulation to survival, J. Control. Release 21, 278-84.

West, G.B., Brown, J.H., Enquist, B.J. (1997). A general model for the origin of 5 allometric scaling laws in biology, Sci. 276, 122-126. 


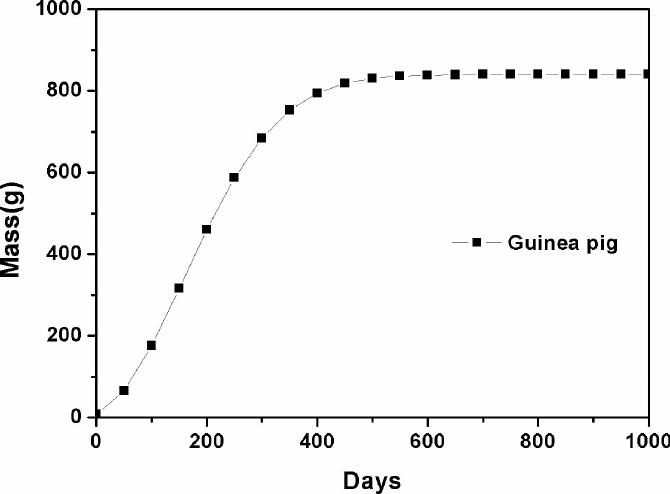




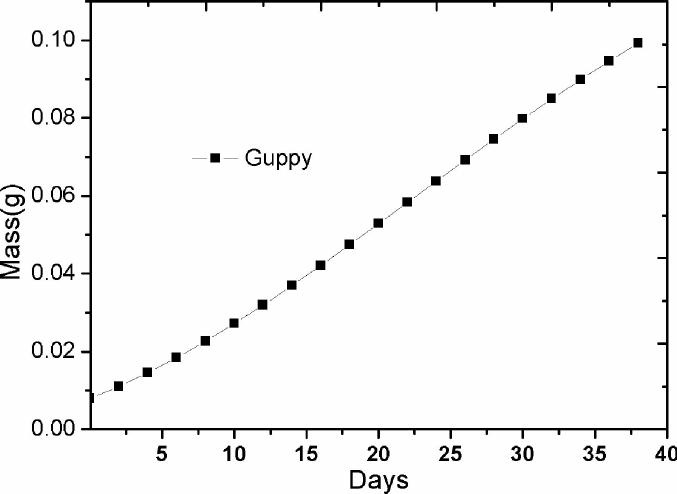




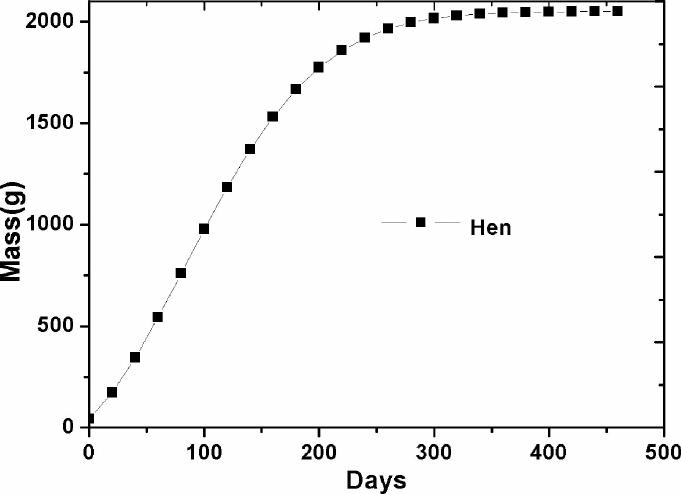




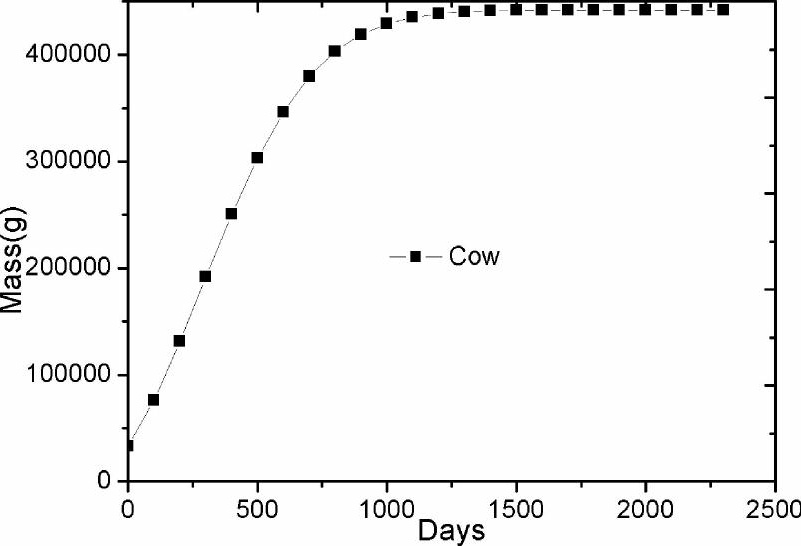

